\title{
Tenotomia do tríceps braquial na reparação das fraturas intercondilares em cães
}

\section{Intercondylar fracture repair following tenotomy of the triceps brachii in the dog}

\author{
André Luis Selmi, ${ }^{*}$ João Guilherme Padilha Filho, ${ }^{\star \star}$ Hélia Cristine Dórea, ${ }^{\star \star \star}$ \\ Júlio Carlos Canola, ${ }^{\star \star}$ Valéria Lima de Sousa ${ }^{\star \star \star \star}$
}

\section{Resumo}

Foram avaliados os resultados clínicos e a técnica de abordagem por meio de tenotomia do músculo tríceps braquial para o tratamento de fraturas epifisárias distais do úmero em 19 cães. Vinte fraturas foram classificadas segundo a apresentação radiográfica. Seis eram fraturas do tipo I, cinco do tipo II, quatro do tipo III e cinco do tipo IV. Foram aplicados pinos de "Rush" e parafusos intercondilares em 12 casos (quatro fraturas tipo I, quatro tipo II, uma tipo III e três tipo IV). Foi utilizado parafuso intercondilar associado a pino de "Steinman" cruzados em forma de X em oito casos, sendo duas fraturas do tipo I, uma do tipo II, três do tipo III e duas do tipo IV. Os animais apresentaram desconforto pós-operatório imediato, regredindo após curto período. Três animais apresentaram seroma até três dias pós-operatórios. As complicações observadas foram a fratura ou deformidade do implante metálico em dois casos e migração dos implantes metálicos em outros dois cães. Obtiveram-se resultados excelentes em 12 membros, bons em quatro, regular em três e um resultado ruim. Pode-se concluir que a abordagem por meio de tenotomia do tríceps braquial propiciou adequada exposição óssea, apresentando complicações comuns a outras abordagens, servindo como mais uma alternativa de acesso cirúrgico à epífise distal do úmero em cães.

Palavras-chave: tenotomia, fraturas epifisárias, úmero, cães.

\begin{abstract}
Clinical results and the surgical approach by means of tenotomy of the triceps brachii for repair of distal epiphyseal fractures of the humerus in 19 dogs were evaluated. Twenty fractures were classified according to its radiographic aspect. Six were a type I fracture, five, a type II, four, a type III and five, a type IV fracture. "Rush". pins and intercondylar screws were used in 12 cases (4 type I, 4 type II, 1 type III and 3 type IV fractures). Crossed "Steinman" pins and intercondylar screws were used in eight fractures, where two were type I, one type II, three type III and two type IV. Dogs exhibited signs of discomfort postoperatively which disappeared within few days after surgery. Three dogs presented seroma formation until three days after surgery. Complications included fracture or deformity of the metallic implant in two dogs and pin migration in other two animals. Excellent results were observed in 12 dogs, good in four, satisfactory in three and one unsatisfactory result. It is concluded that the surgical approach by means of tenotomy of the triceps brachii allowed adequate exposure of the fractured fragments sharing complications with other surgical approaches, and could be considered as an alternative approach for the distal humerus epiphyses.
\end{abstract}

Keywords: tenotomy, epiphyseal fractures, humerus, dogs.

\section{Introdução}

As fraturas intercondilares podem ser consideradas como combinação entre fratura condilar e supracondilar (OImstead, 1995), com baixa incidência, tanto nos homens como nos cães (Anderson et al., 1990), sendo nestes últimos, freqüentemente observadas em adultos, como decorrência de traumatismo (Payne-Johnson e Lewis, 1981; Brinker et al.,
1997). São fraturas que ocorrem com maior incidência na porção articular distal do úmero e que apresentam o pior prognóstico (Anderson et al., 1990; Gupta, 1996; Fornasiéri et al., 1997), sendo fundamental a perfeita redução das superfícies articulares fraturadas, com fixação rígida ininterrupta, permitindo o movimento articular, de forma a se obter resultados funcionais satisfatórios (Lansinger e Máre, 1982; Wang et al., 1994; Turner, 1995; Gupta, 1996; Brinker et al., 1997; Safran et

\footnotetext{
* Pós-Graduando do Curso de Doutorado em Cirurgia Veterinária. Universidade Estadual Paulista/UNESP. Faculdade de Ciências Agrárias e Veterinárias de Jaboticabal/FCAVJ. E-mail:selmi@ fcav.unesp.br

** Professor Assistente. Universidade Estadual Paulista/UNESP, Faculdade de Ciências Agrárias e Veterinárias de Jaboticabal/FCAVJ.

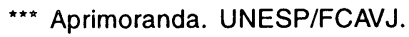

**** Pós-graduanda. Universidade de São Paulo, Faculdade de Medicina Veterinária e Zootecnia.
} 
al., 1999). Metade das fraturas umerais que afetam cães e gatos ocorrem no úmero distal e $74 \%$ dessas envolvem a articulação do cotovelo (Vannini et al., 1988a).

Métodos de fixação, adequado reparo da fratura, tipos de fratura e grau de cominuição são fatores importantes para determinar o prognóstico (Vannini et al., 1988c). Em humanos, o método de osteossíntese para fraturas intercondilares do úmero consiste no uso da placa óssea (Fornasiéri et al., 1997). A ocorrência de doença articular degenerativa após a fratura é inevitável, sendo a reconstituição das superfícies articulares a solução para minimizar este processo (Anderson et al., 1990). A redução imperfeita da fratura pode levar ao desenvolvimento de degeneração e desgaste anormal da articulação (PayneJohnson e Lewis, 1981; Anderson et al., 1990; Brinker et al., 1997), além de anormalidades de ambulação. Devido a isso, a redução fechada com fixação externa tem poucas chances de sucesso (Payne-Johnson e Lewis, 1981; Lansinger e Máre, 1982; Gupta, 1996). Várias técnicas para reparo das fraturas intercondilares em cães foram propostas, como pino transverso aos côndilos, pinos de "Rush", parafuso com compressão interfragmentar através dos côndilos e fio de "Kirshner" (PayneJohnson e Lewis, 1981). Fornasiéri et al. (1997) afirmaram que osteossíntese ideal das fraturas da porção distal do úmero não existe, pois o osso sofre grande tensão cíclica crânio-caudal durante a flexão do cotovelo.

A adequada exposição dos côndilos e a perfeita redução anatômica das superfícies articulares fraturadas foram conseguidas por meio do acesso caudal ao cotovelo (Piermatei e Greeley, 1988; Anderson et al., 1990; Denny, 1993; Olmstead, 1995; Turner, 1995; Brinker et al., 1997). Comumente, as fraturas intercondilares são abordadas através da osteotomia do olécrano (Piermatei e Greeley, 1988; Vannini et al., 1988c; Anderson et al., 1990; Denny, 1993; Olmstead, 1995; Turner, 1995; Brinker et al., 1997), sendo somente feita a tenotomia do tríceps braquial em animais jovens, a fim de se evitar traumatismos à fise ulnar proximal (Vannini et al., 1988b; Olmstead, 1995). A tenotomia do tríceps foi descrita por Dueland (1974) no tratamento de fraturas envolvendo a epífise distal do úmero, e posteriormente por Anderson et al. (1989) para acesso ao cotovelo no tratamento cirúrgico da fragmentação do processo coronóide e da osteocondrite dissecante.

O presente estudo objetivou avaliar os resultados pós-operatórios e a técnica de abordagem por meio de tenotomia do músculo tríceps braquial em cães adultos portadores de fraturas epifisárias distais do úmero.

\section{Material e métodos}

Foram atendidos 19 animais no Serviço de Ortopedia e Traumatologia do Hospital Veterinário da UNESP/ Campus de Jaboticabal apresentando fratura intercondilar umeral, no período compreendido entre janeiro de 1996 e janeiro de 2000.

Os dados referentes a idade, sexo, peso, raça, tipo de traumatismo, membro afetado, tipos de fratura, histórico do animal e problemas concomitantes foram obtidos a partir das fichas clínicas dos animais. Os animais cujas fichas não continham toda informação necessária para participação neste estudo, não foram incluídos. Os animais foram radiografados nas posições médio-lateral e crânio-caudal do cotovelo, sob anestesia geral empregando-se levomepromazina ${ }^{a}$ $(0,5 \mathrm{mg} / \mathrm{kg})$ seguida de propofol, ${ }^{\mathrm{b}}$ na dose média de $5 \mathrm{mg} / \mathrm{kg}$, ambos por via intravenosa, para caracterizar o tipo de fratura e definir o melhor procedimento de osteossíntese.

As fraturas foram classificadas segundo o sistema descrito por Bardet et al. (1983) como: tipo I (associação de fratura supracondilar medial transversa com supracondilar lateral em bizel e fratura intercondilar), tipo II (associação de fratura supracondilar medial em bizel com supracondilar lateral transversa e fratura intercondilar), tipo III (fratura supracondilar lateral e medial transversa com fratura intercondilar), tipo IV (fratura supracondilar medial e lateral cominutivas com fratura intercondilar) e tipo $\mathrm{V}$ (fratura supracondilar medial e lateral cominutivas com fratura intercondilar cominutiva).

Todos os animais foram submetidos a exames hematológicos pré-operatórios, que consistiam na realização de hemoleucograma, contagem de plaquetas e dosagem sérica de creatinina, uréia e alaninamino-transferase. Cefazolina sódicac $(20 \mathrm{mg} / \mathrm{kg})$ e cetoprofeno $(2 \mathrm{mg} / \mathrm{kg})$ foram administrados por via endovenosa, uma hora previamente à cirurgia, sendo a terapia antiinflamatória estendida por três dias após a cirurgia. Os animais aqui estudados tiveram a sensibilidade distal do membro testada por meio de pinçamento digital no momento da primeira consulta e no primeiro dia pós-operatório, para efeito de documentação da integridade do nervo radial, uma vez que fraturas distais do úmero podem estar acompanhadas de lesão a esta estrutura com agravamento do prognóstico (Vannini et al., 1988c).

Os animais foram pré-medicados com a associação de cloridrato de meperidina ${ }^{e}(5 \mathrm{mg} / \mathrm{kg})$ e cloridrato de levomepromazina $(0,5 \mathrm{mg} / \mathrm{kg})$, ambos por via intramuscular. Após 15 minutos, a anestesia foi induzida com propofol (dose média de $5 \mathrm{mg} / \mathrm{kg}$ ), por via intravenosa, e, ato contínuo, era realizada a intubação orotraqueal com sonda provida de balão. A anestesia cirúrgica foi mantida em todos os animais por meio da administração de sevoflurano' diluído em oxigênio a $100 \%$, empregando-se circuito semi-fechado.

Para abordagem ao foco de fratura, os cães foram posicionados em decúbito dorsal, e o membro afetado foi preparado assepticamente para cirurgia. Realizou-se incisão cutânea longitudinal, no aspecto caudo-lateral da articulação do cotovelo, que se estendia desde o terço distal do úmero, até o terço proximal da ulna, cruzando a articulação entre o olécrano e o côndilo lateral. $O$ tecido subcutâneo foi divulsionado seguido da incisão da fáscia profunda, tanto medial como lateralmente, e a fáscia localizada ao lado da borda cranial da cabeça medial do tríceps, incisada. O nervo ulnar foi identificado e afastado, assim como os vasos ulnares colaterais. A margem cranial da cabeça lateral do tríceps foi também separada de sua junção com a fáscia. Desta forma obteve-se exposição do olécrano, da inserção do tríceps e epicôndilos do úmero. A tenotomia do tríceps foi executada imediatamen-

\footnotetext{
a Cloridrato de Levomepromazina. Neozine. Rhodia-Poulenc.

b Propofol. Diprivan. Zeneca do Brasil.

c Cefazolina sódica. Kefazol. Elli-Lilly

d Cetoprofeno. Ketofen 1\% - Rhodia-Poulenc.

- Cloridrato de Meperidina. Dolossal. Hoechst do Brasil.

' Sevoflurano. Sevorane. Abbott do Brasil.
} 
te proximal ao olécrano, deixando-se pequena margem de tendão para posterior tenorrafia, obtendo-se assim, perfeita exposição da fossa do olécrano e porção interna e caudal dos côndilos umerais.

O tratamento cirúrgico foi baseado na redução e fixação da fratura com implantes metálicos, incluindo parafusos intercondilares com efeito de compressão interfragmentar, pinos de "Rush" e pinos de "Steinman", cujo diâmetro variou de acordo com o peso e porte do animal.

Após a síntese da fratura, procedeu-se à tenorrafia do tríceps adotando-se técnica de polia de três alças (Berg e Egger, 1986) associado à sutura epitendínea com pontos interrompidos simples, usando fio de sutura inabsorvível sintético, tipo poliamida $2-0^{9}$. As fáscias musculares e o tecido subcutâneo foram aproximadas empregando-se fio de poliglactina $910^{\text {h }}$ em padrão de sutura contínuo simples. A pele foi suturada empregando-se fio de poliamida em padrão isolado simples. Não se realizou qualquer tipo de imobilização externa após o ato cirúrgico em nenhum dos animais.

Os animais foram mantidos em repouso, para que suas atividades ficassem restritas, durante duas semanas subseqüentes à cirurgia. As atividades normais dos animais só puderam retornar, após a consolidação da fratura, constatada radiograficamente. $\mathrm{O}$ acompanhamento pós-operatório dos animais se deu num período entre um e 12 meses após a cirurgia.

A função do membro operado foi classificada de acordo com Bardet et al. (1983) em: excelente (aparentemente função normal do membro, sem claudicação ou limitação da amplitude articular), boa (atividade normal com sustentação do peso corporal, a maior parte do tempo, leve limitação da amplitude articular após repouso prolongado ou exercício intenso), satisfatória (membro funcional mas com claudicação freqüente) e ruim (membro afuncional ou claudicação severa).

\section{Resultados}

Dentre os 19 animais, foram observadas o total de 20 fraturas, onde 13 animais eram machos e seis fêmeas, com idade variando entre um e sete anos de idade. Foram observados 12 animais sem raça definida, seguidos por um Poodle, um Cocker Spaniel Inglês, um Pastor Alemão, um Terrier Brasileiro, um Pinscher, um Retriever do Labrador e um Jack Russel Terrier. A maioria dos animais apresentou fratura após atropelamento (8/19), apesar de muitos proprietários não saberem o motivo do trauma de seus animais (6/19), que desapareceram e depois retornaram com o problema. Outras causas incluiam briga e queda. Onze animais apresentaram fratura intercondilar do úmero direito e sete, do úmero esquerdo. Um animal apresentou fratura intercondilar umeral bilateral.

Os pacientes com menos de um ano de idade foram considerados jovens enquanto aqueles com mais de um ano foram considerados adultos; desta forma, todas as fraturas nesse estudo ocorreram em animais adultos.

Foram observadas seis fraturas intercondilares umeral do tipo I, cinco do tipo II, quatro do tipo III e cinco do tipo IV. Foram

\footnotetext{
g Poliamida. Mononylon. Ethicon.

n Poliglactina 910. Vycril. Ethicon.
}

aplicados pinos de "Rush" e parafusos intercondilares em 12 casos (quatro fraturas tipo I, quatro tipo II, uma tipo III e três tipo IV). Foi utilizado parafuso intercondilar associado a pino de "Steinman" cruzados em forma de X em oito casos, sendo duas fraturas do tipo I, uma do tipo II, três do tipo III e duas do tipo IV. O tempo entre o momento da fratura e o tratamento cirúrgico variou entre um e vinte e dois dias, sendo que a maioria das fraturas (15/20) foram reparadas dentro de cinco dias após a ocorrência. Cinco fraturas, sendo quatro do tipo II e uma do tipo I, foram tratadas com mais de dez dias depois do acidente.

Imediatamente após a cirurgia, os animais não utilizavam o membro operado e evitavam a manipulação passiva, dificultando o trabalho de fisioterapia, que consistia na realização de exercícios de extensão e flexão articular para manutenção da amplitude articular. Progressivamente permitiam a manipulação do membro operado, inicialmente com pequena amplitude de movimento e por entre o quarto e oitavo dia pósoperatório, com total movimento da articulação. Após o quarto pós-operatório, os animais começaram a apoiar levemente o membro operado, ainda apresentando claudicação, até que, por volta do trigésimo dia após a cirurgia, os cães demonstravam ambulação satisfatória.

As complicações observadas incluíram fratura ou deformidade do implante metálico em dois casos, e migração dos pinos de "Rush" e "Steinman" em outros dois casos em direção ao ponto de penetração óssea, obrigando sua retirada após a consolidação da fratura. Em três destes casos, a osteossíntese foi refeita e novos implantes metálicos foram empregados, porém os animais mantiveram claudicação freqüente. Em um cão, devido ao avançado grau de degeneração articular, optou-se pela artrodese da articulação úmerorádio-ulnar, o que limitou a amplitude de movimento deste membro.

Três outros animais apresentaram seroma no membro operado entre três e cinco dias após a cirurgia, que foi drenado com agulha fina e o membro foi imobilizado por meio de faixa compressiva por três dias, a fim de se evitar a progressão do processo.

Foram obtidos excelentes resultados em $60 \%$ dos membros operados, bons em $20 \%$, regulares em $15 \%$ e apenas um resultado ruim (5\%). Em cada retorno ou contato telefônico com o proprietário, este era questionado sobre os efeitos da cirurgia na atividade ambulatória de seu animal.

\section{Discussão}

Neste trabalho, a maioria das fraturas foi gerada por atropelamento, seguida por queda, briga ou causas deconhecidas, concordando com as citações de Payne-Johnson e Lewis, 1981; Lansinger e Máre, 1982; Anderson et al., 1989; Anderson et al., 1990; Denny, 1993; Olmstead, 1995; Turner, 1995; Gupta, 1996; Fornasiéri et al. (1997), já que as fraturas envolvendo a epífise distal do úmero resultam do trauma direcionado dorsalmente através da cabeça do rádio à tróclea umeral. Não foi observada nenhuma prevalência significativa entre os membros torácicos fraturados, sendo 12 fraturas do úmero direito e oito do úmero esquerdo, assim como observado por Anderson et al. (1990). Observou-se que fraturas intercondilares umerais acometem principalmente animais adultos, fato já descrito por Anderson et al. (1990) e Brinker et al. (1997). 
A escolha pelo método de classificação das fraturas descrito por Bardet et al. (1983) permitiu planejamento do método de fixação das fraturas e análise retrospectiva detalhada.

De acordo com Vannini et al. (1988c), fraturas condilares ou supracondilares são freqüentemente acompanhadas de lesão nervosa envolvendo o nervo radial, podendo ocorrer trauma iatrogênico durante $O$ ato cirúrgico, tanto ao nervo radial quanto ao nervo ulnar. A técnica de tenotomia do tríceps para abordagem ao cotovelo permitiu boa visualização do nervo ulnar, que é afastado durante todo o procedimento cirúrgico, evitando, desta forma, lesões nervosas. Esta abordagem propicia ainda manipulação distante do trajeto do nervo radial sobre o aspecto lateral da epífise distal do úmero, minimizando lesões nervosas iatrogênicas, conforme observações de Dueland (1974).

O acesso caudal ao cotovelo tem maior aceitação de acordo com Payne-Johnson e Lewis (1981), Anderson et al. (1989), Anderson et al. (1990), Denny (1993), Olmstead (1995), Gupta (1996), Brinker et al. (1997) e Fornasiéri et al. (1997). Dueland (1974), Vannini et al. (1988b) e Olmstead (1995) empregaram a tenotomia do tríceps para abordagem ao cotovelo em cães. Corroborando as citações de Dueland (1974), esta abordagem permitiu boa exposição dos côndilos umerais, possibilitando perfeita redução das superfícies articulares fraturadas, e facilitou a osteossintese com menor trauma cirúrgico, em relação à técnica de osteotomia do olécrano empregada por Payne-Johnson e Lewis (1981), Anderson et al. (1989), Anderson et al. (1990), Denny (1993), Olmstead (1995), Gupta (1996) e Brinker et al. (1997). Apesar de não ser objeto deste estudo, observou-se também que a rafia tendínea pela técnica citada por Berg e Egger (1986) favoreceu aposição adequada dos cotos tendíneos sem complicações pós-operatórias relacionadas diretamente com a técnica, corroborando com a suposição de que esta técnica de tenorrafia exige menor suporte externo durante os estágios iniciais de cicatrização tendínea. $O$ posicionamento do paciente em decúbito dorsal no transoperatório, possibilitou adequado acesso cirúrgico, facilitando a manipulação dos fragmentos ósseos pelo cirurgião.

A base da osteossíntese neste estudo foi o uso de parafusos intercondilares com compressão interfragmentar, combinados com pinos de "Rush" e pinos de "Steinman", obtendo-se estabilidade necessária para consolidação da fratura sem grande exposição óssea, contrariando as citações de Anderson et al. (1990) e Fornasiéri et al. (1997), que optaram pelo uso de placas ósseas para o reparo das fraturas intercondilares. Vannini et al. (1988c) utilizaram parafusos associados a pinos em pacientes com menos de um ano de idade, preferindo a utilização de placas ósseas na reparação de fraturas supracondilares e dicondilares. Tal opinião também é compartilhada por Anderson et al. (1990) que associaram a compressão interfragmentar por parafuso a placas ósseas em fraturas supracondilares associadas a fraturas intercondilares. Na opinião desses autores, a utilização de parafusos intercondilares associados a pinos intramedulares possibilitou estabilidade adequada ao foco de fratura, inclusive quando a cominuição óssea supracondilar era de pequena extensão, possibilitando, assim, ambulação e apoio do membro operado, desde que respeitada superfície de con- tato suficiente entre o fragmento distal e o proximal, de modo que houvesse distribuição de cargas entre os fragmentos ósseos e os implantes. Outrossim, nos casos onde foram observados fratura ou deformidade do implante, observou-se cominuição óssea sem justaposição dos fragmentos após a síntese, de forma que os implantes metálicos ficaram sujeitos a maiores cargas biomecânicas, fato que foi contornado pela aplicação de pinos de maior calibre em um animal, e artrodese em um segundo cão que apresentava imagens radiográficas sugestivas de osteoartrose severa, impossibilitando uma segunda tentativa de síntese.

Comparando nossos resultados com aqueles de Anderson et al. (1990), podemos inferir que o índice de casos com resolução excelente ou boa foi similar. As complicações observadas em nosso estudo, que incluíram fratura do implante em dois cães (fratura óssea tipo IV) e migração destes em outros dois (fraturas do tipo II e III, respectivamente) produziram resultados satisfatórios em três animais e ruim em um. Denny (1993), utilizando-se de placas e parafusos no tratamento de fraturas intercondilares, obteve resultados satisfatórios em $46 \%$ dos casos, enquanto que pinos intramedulares associados a parafusos intercondilares apresentaram menor índice de complicações.

Outras complicações, como a formação de seroma em três cães, podem estar relacionadas com a divulsão de tecidos moles, falta de eliminação de espaço morto durante a síntese destes tecidos ou ao excesso de atividade pós-operatória; porém, em nenhum dos casos, esta manifestação evoluiu para complicações mais severas, sendo portanto adequada a drenagem associada à compressão para a satisfatória resolução do problema. Dueland (1974) descreveu que as complicações decorrentes da abordagem por tenotomia do tríceps estavam relacionadas com a tensão na linha de sutura e viabilidade tendínea, e em seu estudo não observou formação de seroma devido ao emprego de drenagem ativa no pósoperatório.

O início da movimentação passiva do membro operado, logo após a cirurgia, proporcionou boa e precoce recuperação das funções normais do cotovelo acometido, indo de acordo com Dueland (1974) e Anderson et al. (1990), e Safran et al. (1999), que indicaram os benefícios da fisioterapia pós-operatória na recuperação de cães e humanos, respectivamente, já que - retorno precoce das funções locomotoras previne a anquilose, assim como doenças degenerativas (PayneJohnson e Lewis, 1981; Olmstead 1995).

\section{Conclusão}

O emprego da técnica de tenotomia do tríceps para abordagem ao cotovelo propicia vantagens no transoperatório, permitindo a perfeita exposição dos côndilos e visualização precisa dos contornos articulares, sem técnica cruenta de osteotomia, facilitando o trabalho de fixação da fratura. No pós-operatório, permite que $o$ animal recupere precocemente as funções normais do membro operado, com baixo índice de complicações. Ainda, a isenção de implante metálico para osteossíntese do olécrano reduz os custos e tempo do procedimento cirúrgico, podendo ser indicado como uma alternativa à abordagem dos côndilos umerais em animais adultos. 


\section{Referências}

ANDERSON, S.M., LIPPINCOTT, C.L., SCHULMAN, A.J. Longitudinal myotomy of flexor carpi radialis: A new approach for the medial aspect of the elbow.joint. Journal of the American Animal Hospital Association. v. 25, n. 5 , p. 499-503, 1989.

ANDERSON, T.J., CHARMICHAEL, S., MILLER, A. Intercondylar humeral fracture in the dog - a review of 20 cases. Journal of Small Animal Practice. v. 31, p. 437-442, 1990.

BARDET, J. F., HOHNR.B., RUDY, R.L., et al. Fractures of the humerus in dogs and cats: a retrospective study of 130 cases. Veterinary Surgery. v. 12, p. 73-77, 1983.

BERG, R.J., EGGER, E.L. In vitro comparison of the three-loop pulley and locking loop sutures patterns for repair of the canine weightbearing tendons and collateral ligaments. Veterinary Surgery. v. 15, p. 107-110, 1986.

BRINKER, W.O., PIERMATEI, D.C., FLO, G.L. Handbook of small animal orthopedics and fracture repair. 3. ed. Philadelphia: Saunders, 1997. Cap. 10: Fractures of the humerus: p. 285-287.

DENNY, H.R. A guide to canine and feline orthopaedic surgery. 3. ed. London: Blackwell Scientific Publications, 1993, p. 224-226.

DUELAND, $R$. Triceps tenotomy approach for distal fractures of the canine humerus. Journal of the American Veterinary Medical Association. v. 165, n. 1, p. 82-86, 1974.

FORNASIÉRI, C., STAUB, C., TOURNÉ, Y., et al. Biomechanical comparative study of three types of osteosynthesis in the treatment of supra and intercondylar fractures of the humerus in adults. Revue de Chirurgie Ortopedique et Reparatrice de L 'appareil Moteur. v. 83, n. 3, p. 237-242, 1997.

GUPTA, R. Intercondylar fractures of the distal humerus in adults. Injury. v. 27, n. 8, p. 569-572, 1996.
LANSINGER, O., MÁRE, K. Intercondylar T-fractures of the humerus in adults. Archives of Orthopedic and Trauma Surgery. v. 100, n. 1, p. 3742, 1982.

OLMSTEAD, M.L. Fraturas do úmero. In: SLATER, D.H. Manual de cirurgia de pequenos animais, 2. ed. Philadelphia: Saunders, 1995. Cap. 130. p. 2033-2047.

PAYNE-JOHNSON, M., LEWIS, D.G. A technique for fixation of intercondylar humeral fractures in immature small dogs. Journal of Small Animal Practice. v. 22, p. 293-299, 1981.

PIERMATEI D.L., GREELEY R.G. Atlas de abordagens cirúrgicas aos ossos do cão e do gato, 2. ed. Philadelphia: Saunders, 1988. p. 92-95.

SAFRAN, O., MOSHEIFF, R., SEGAL, D., et al. Surgical treatment of intercondylar fractures of the humerus in adults. American Journal of Orthopedics. v. 28, n. 11, p. 659-662, 1999.

TURNER, T.M. Fractures of the bones of the front limb. In: OLMSTEAD, M.L. Small animal orthopedics, St Louis: Mosby-Year Book. 1995. Cap. 8. p. 195-217.

VANNINI, R., OLMSTEAD, M.L., SMEAK D.D (a). An epidemiological study of 151 distal humeral fractures in dogs and cats. Journal of the American Animal Hospital Association. v. 24, p. 531-536, 1988.

VANNINI, R., OLMSTEAD, M.L., SMEAK D.D. (b). Humeral condylar fractures caused by minor trauma in 20 adult dogs. Journal of the American Animal Hospital Association. v. 24, p. 355-362, 1988.

VANNINI, R., SMEAKD.D., OLMSTEAD, M.L. (c). Evaluation of surgical repair of 135 distal humeral fractures in dogs and cats. Journal of the American Animal Hospital Association. v. 24, p. 537-545, 1988.

WANG, K.C., SHIH, I.I.N., ILSU, K.Y. et al. Intercondylar fractures of the distal humerus: routine anterior subcutaneous transposition of the ulnar nerve in a posterior operative approach. Journal of Trauma. $v$. 36, n. 6, p. $770-773,1994$. 